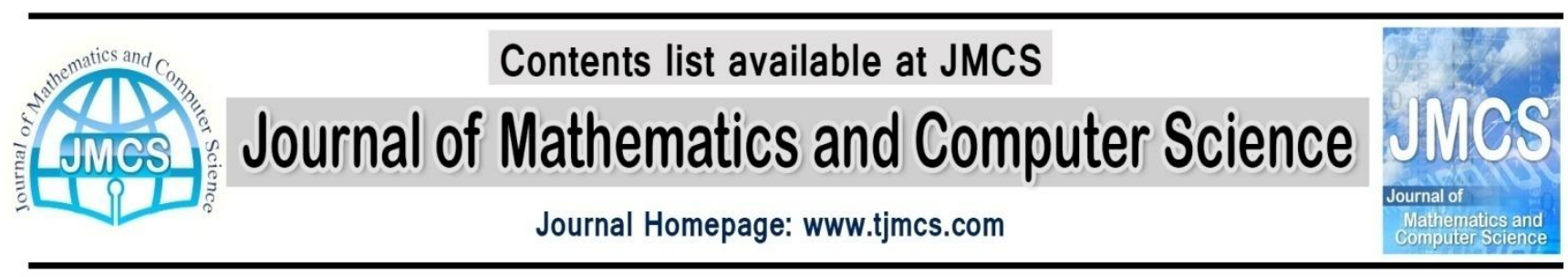

\title{
The Numerical Solution of Nonlinear Fredholm-Hammerstein Integral Equations of the Second Kind Utilizing Chebyshev Wavelets
}

\author{
M. M. Shamooshaky ${ }^{1}$, P. Assari ${ }^{2}$, H. Adibi ${ }^{2, *}$ \\ ${ }^{I}$ Department of Mathematics, Imam Hossein University, P.O. Box 16895-198, Tehran, Iran \\ ${ }^{2}$ Department of Applied Mathematics, Faculty of Mathematics and Computer Science, \\ Amirkabir University of Technology, No. 424, Hafez Ave., Tehran 15914, Iran \\ *adibih@aut.ac.ir
}

\begin{abstract}
Article history:
Received March 2014

Accepted April 2014

Available online May 2014
\end{abstract}

\begin{abstract}
This paper describes a numerical scheme based on the Chebyshev wavelets constructed on the unit interval and the Galerkin method for solving nonlinear Fredholm-Hammerstein integral equations of the second kind. Chebyshev wavelets, as very well localized functions, are considerably effective to estimate an unknown function. The integrals included in the method developed in the current paper are approximated by the Gauss-Chebyshev quadrature rule. The proposed scheme reduces FredholmHammerstein integral equations to the solution of nonlinear systems of algebraic equations. The properties of Chebyshev wavelets are used to make the wavelet coefficient matrix sparse which eventually leads to the sparsity of the coefficients matrix of obtained system. Some illustrative examples are presented to show the validity and efficiency of the new technique.
\end{abstract}

Keywords: Fredholm-Hammerstein integral equation; Chebyshev wavelet; Galerkin method; GaussChebyshev quadrature rule; sparse matrix

\section{Introduction}

In this paper, we study the nonlinear Fredholm-Hammerstein integral equation of the second kind, namely

$$
u(x)-\lambda \int_{0}^{1} K(x, y) \phi(y, u(y)) \mathrm{d} y=f(x), \quad 0 \leq x \leq 1,
$$

where the right-hand side $f \in L_{w}^{2}[0,1]$ and the kernel $K \in L_{w}^{2}([0,1] \times[0,1])$, with $w(x)=1 / 2 \sqrt{x(1-x)}$, are given functions, $u$ is the unknown function to be determined, $\lambda \in \mathbf{R}$ is a constant and the known function $\phi$ is continuous and nonlinear corresponding to the variable $u$. These types of integral equations arise as 
a reformulation of two-point boundary value problems with a certain nonlinear boundary condition which occur in many problems of mathematical physics, fluid mechanics and electrochemical machining [1,2].

To obtain the numerical solution of these types of integral equations, many different basis functions have been used as basis in the projection methods, including the collocation and Galerkin methods. The main advantage of applying the projection methods is that they reduce an integral equation to the solution of a system of algebraic equations. However, there are so many different families of basic functions which can be used in these methods which it is sometimes difficult to select the most suitable one. Atkinson has applied the piecewise polynomials of order $n$ in the projection methods for the numerical solution of nonlinear Fredholm integral equations and obtained the convergence of polynomial order [3]. Walsh-Hybrid functions have been used to solve Fredholm-Hammerstein integral equation of second kind in [4]. Alipanah and Dehghan [5] have investigated a method for the numerical solution of nonlinear Fredholm-Hammerstein integral equations utilizing the collocation method and positive definite functions. Sinc-collocation method for the solution of nonlinear Fredholm integral equations have been presented in $[6,7]$.

Since 1991, wavelet technique has been applied to solve integral equations [8,9]. The wavelet method allows the creation of very fast algorithms when compared with the algorithms ordinarily used. Wavelets are considerably useful for solving Fredholm-Hammerstein integral equations and provide accurate solutions. B-spline wavelets have been applied to solve Fredholm-Hammerstein integral equations of the second kind in [10]. Lepik [11] has studied a computational method for solving Fredholm-Hammerstein integral equations of the second kind by using Haar wavelets. Authors of [1] have introduced a numerical method for solving nonlinear Hammerstein integral equations utilizing Alpert wavelets as basis in the Petrov-Galerkin method. Legendre wavelets have been used to approximate the solution of linear and nonlinear integral equations with weakly singular kernels in [12].

The purpose of this paper is to present a method of obtaining numerical solutions of Eq. (1) by combining the Chebyshev wavelets and the Galerkin method. The properties of Chebyshev wavelets together with the Gauss-Chebyshev integration method are used to convert Eq. (1) into a nonlinear system of algebraic equations. This system may be solved by using an appropriate numerical method, such as Newtons iteration method. We will notice that, these wavelets make the wavelet coefficient matrices sparse and accordingly it leads to the sparsity of the coefficient matrix of the final system and provide accurate solutions.

Furthermore, the Chebyshev wavelets have been used to approximate the solution of differential equations [13], the second kind integral equations [14], the first kind Fredholm integral equations [15], Abel's integral equations [16], nonlinear systems of Volterra integral equations [17], fractional nonlinear Fredholm integro-differential equations [18], time-varying delay systems [19], fractional differential equations [20] and a nonlinear fractional differential equation [21].

The outline of the paper is as follows: In Section 2, we review some properties of Chebyshev wavelets and approximate the function $f(x)$ by these wavelets. Section 3 is devoted to present a computational method for solving Eq. (1) utilizing Chebyshev wavelets and the error analysis for the method. Numerical examples are given in Section 4. Finally, we conclude the article in Section 5.

\section{Properties of Chebyshev wavelets}

Wavelets consist of a family of functions constructed from dilation and translation of a single function called the mother wavelet. When the dilation parameter $a$ and the translation parameter $b$ vary continuously, we have the following family of continuous wavelets $[8,22]$

$$
\psi_{a, b}(t)=|a|^{\frac{-1}{2}} \psi\left(\frac{t-b}{a}\right), a, b \in \mathbf{R}, a \neq 0 .
$$

If we restrict the parameters $a$ and $b$ to discrete values $a=a_{0}^{-k}, b=n b_{0} a_{0}^{-k}, a_{0}>1, b_{0}>0$ where $n$ and $k$ 
are positive integers, then we have the following family of discrete wavelets

$$
\psi_{k, n}(t)=\left|a_{0}\right|^{\frac{k}{2}} \psi\left(a_{0}^{k} t-n b_{0}\right),
$$

where $\psi_{k, n}(t)$ form a wavelet basis for $L^{2}(\mathbf{R})$. In particular, when $a_{0}=2, b_{0}=1$ then $\psi_{k, n}(t)$ forms an orthonormal basis [8].

\subsection{Chebyshev wavelets}

Chebyshev wavelets, $\psi_{n, m}(t)=\psi(k, n, m, t)$ have four arguments; $n=1,2, \ldots, 2^{k-1}, k$ can assume any nonnegative integer, $m$ is the degree of Chebyshev polynomial of the first kind and $t$ denotes independent variable in $[0,1]$ :

$$
\psi_{n, m}(t)=\left\{\begin{array}{cc}
\alpha_{m} \sqrt{\frac{2^{k}}{\pi}} T_{m}\left(2^{k} t-2 n+1\right), & \frac{n-1}{2^{k-1}} \leq t<\frac{n}{2^{k-1}}, \\
0, & \text { otherwise }
\end{array}\right.
$$

where

$$
\alpha_{m}= \begin{cases}1, & m=0 \\ 2, & m>0\end{cases}
$$

and $m=0,1, \ldots, M-1, n=1,2, \ldots, 2^{k-1}[14]$. Here $T_{m}, m=0,1, \ldots$, are the Chebyshev polynomials of the first kind which are orthogonal with respect to the weight function $w\left(\frac{t+1}{2}\right)=1 / \sqrt{1-t^{2}}$, on the interval $[-1,1]$, and satisfy the following recursive formula:

$$
T_{0}(t)=1, T_{1}(t)=t, T_{m}(t)=2 t T_{m-1}(t)-T_{m-2}(t), m=2,3, \ldots \ldots
$$

For these functions we also have the following useful formula:

$$
T_{m}(\cos \theta)=\cos m \theta, m=0,1,2, \ldots
$$

We should note that Chebyshev wavelets are orthonormal set with respect to the weight function

$$
w_{k}(t)=\left\{\begin{array}{cc}
w_{1, k}(t), & 0 \leq t<\frac{1}{2^{k-1}}, \\
w_{2, k}(t), & \frac{1}{2^{k-1}} \leq t<\frac{2}{2^{k-1}}, \\
\vdots & \vdots \\
w_{2^{k-1}, k}(t), & \frac{2^{k-1}-1}{2^{k-1}} \leq t<1,
\end{array}\right.
$$

where $w_{n, k}(t)=w\left(2^{k-1} t-n+1\right)$.

\subsection{Function approximation}

A function $f(x) \in L_{w_{k}}^{2}[0,1]$, may be expanded as 


$$
f(x)=\sum_{n=1}^{\infty} \sum_{m=0}^{\infty} c_{n, m} \psi_{n, m}(x)
$$

where

$$
c_{n, m}=<f(x), \psi_{n, m}(x)>_{w_{k}},
$$

in which $<., .>_{w_{k}}$ denotes the inner product in $L_{w_{k}}^{2}[0,1]$. The series (7) is truncated as

$$
f(x) \approx P_{k, M} f(x)=\sum_{n=1}^{2^{k-1}} \sum_{m=0}^{M-1} c_{n, m} \psi_{n, m}(x)=C^{T} \Psi(x),
$$

where $C$ and $\Psi$ are $2^{k-1} M$ vectors given by

$$
\begin{gathered}
C=\left[c_{1,0}, c_{1,1}, \ldots, c_{1, M-1}, c_{2,0}, c_{2,0}, \ldots, c_{2, M-1}, \ldots, c_{2^{k-1}, 0}, \ldots, c_{2^{k-1}, M-1}\right]^{T} \\
=\left[c_{1}, c_{2}, \ldots, c_{2^{k-1} M}\right]^{T},
\end{gathered}
$$

and

$$
\begin{gathered}
\Psi=\left[\psi_{1,0}, \psi_{1,1}, \ldots, \psi_{1, M-1}, \psi_{2,0}, \psi_{2,1}, \ldots, \psi_{2, M-1}, \ldots, \psi_{2^{k-1}, 0}, \ldots, \psi_{2^{k-1}, M-1}\right]^{T} \\
=\left[\psi_{1}, \psi_{2}, \ldots, \psi_{2^{k-1} M}\right]^{T} .
\end{gathered}
$$

Based on the above formulations, we can present the following theorem from [15]:

Theorem 2.1.A function $f(x) \in L_{w_{k}}^{2}[0,1]$, with bounded second derivative, say $\left|f^{\prime \prime}(x)\right| \leq \gamma$, canbe expanded as an infinite sum of the Chebyshev wavelets, and the series converges uniformly to $f(x)$, that is

$$
f(x)=\sum_{n=1}^{\infty} \sum_{m=0}^{\infty} c_{n, m} \psi_{n, m}(x)
$$

furthermore, we have

$$
\left\|P_{k, M} f-f\right\|_{\infty} \leq \frac{\sqrt{\pi} \gamma}{2} \sum_{n=2^{k-1}+1}^{\infty} \sum_{m=M}^{\infty} \frac{1}{n^{\frac{5}{2}}\left(m^{2}-1\right)}, x \in[0,1] .
$$

Considering $i=M(n-1)+m+1$ and $j=M\left(n^{\prime}-1\right) m^{\prime}+1$, we approximate $K(x, y) \in L_{w_{k}}^{2}([0,1] \times[0,1])$ as

$$
K(x, y) \approx \sum_{i=1}^{2^{k-1} M} \sum_{j=1}^{2^{k-1} M} K_{i j} \psi_{i}(x) \psi_{j}(y)=\Psi^{T}(x) \mathbf{K} \Psi(y),
$$

where $\mathbf{K}=\left[K_{i j}\right]_{1 \leq i, j \leq 2^{k-1} M}$ with the entries

$$
K_{i j}=<\psi_{i}(x),<K(x, y), \psi_{j}(y)>_{w_{k}}>_{w_{k}} .
$$

\section{Solution of Hammerstein integral equations}

In this section, the Chebyshev wavelet method is used for solving Fredholm-Hammerstein integral equations of the second kind in the form Eq. (1). For this aim, we let

$$
z(x)=\phi(x, u(x))
$$


and approximate functions $f(x), u(y), Z(x)$ and $K(x, y)$ in the matrix forms

$$
\begin{gathered}
f(x) \approx F^{t} \Psi(x), \\
u(x) \approx U^{t} \Psi(x), \\
z(x) \approx Z^{t} \Psi(x), \\
K(x, y) \approx \Psi^{t}(x) \mathbf{K} \Psi(y) .
\end{gathered}
$$

By substituting (16)-(19) into (1), we obtain

$$
\begin{aligned}
\Psi^{t}(x) U & =\lambda \int_{0}^{1} \Psi^{t}(x) \mathbf{K} \Psi(y) \Psi^{t}(y) Z \mathrm{~d} y+\Psi^{t}(x) F \\
& =\lambda \Psi^{t}(x) \mathbf{K}\left(\int_{0}^{1} \Psi(y) \Psi^{t}(y) \mathrm{d} y\right) Z+\Psi^{t}(x) F .
\end{aligned}
$$

By letting

$$
\int_{0}^{1} \Psi(y) \Psi^{t}(y) \mathrm{d} y=\mathbf{L}
$$

where $\mathbf{L}$ is a $2^{k-1} M \times 2^{k-1} M$ matrix which is computed next. So, we have

$$
\Psi^{t}(x) U-\lambda \Psi^{t}(x) \mathbf{K} \mathbf{L} Z=\Psi^{t}(x) F .
$$

Now, based on the Galarkin method, by taking inner product $<\Psi(x), .>$ upon both sides of Eq. (23) and using Eq. (22) we obtain

$$
U=\lambda \mathbf{K} Z+F
$$

Thus, we have

$$
u(x) \approx \Psi^{t}(x)(\lambda \mathbf{K} Z+F) .
$$

By substituting (18) and (25) in Eq. (15), we get

$$
\Psi^{t}(x) Z=\phi\left(x, \Psi^{t}(x)(\lambda \mathbf{K} Z+F)\right) .
$$

By evaluating Eq. (26) at collocation points $\left\{x_{j}^{\prime}\right\}_{j=1}^{2^{k}(2 M+1)}$, where $x_{j}^{\prime}=\frac{\left(j-\frac{1}{2}\right)}{2^{k}(2 M+1)}$, we obtain the following nonlinear system of algebraic equations

$$
\Psi^{t}\left(x_{j}^{\prime}\right) Z=\phi\left(x_{j}^{\prime}, \Psi^{t}\left(x_{j}^{\prime}\right)(\lambda \mathbf{K} Z+F)\right), \quad j=1,2, \ldots, 2^{k}(2 M+1),
$$

for unknowns $Z=\left[z_{1}, z_{2}, \ldots, z_{2^{k}(2 M+1)}\right]^{t}$. Then, by computing $Z$, we can obtain the vector $U$ from (24) and acquire the approximate solution of Eq. (1), by using (17).

Remark 1.Analytical calculation of the matrixes $F$ and $K$ is usually difficult and takes considerable times. Therefore, it is required to utilize an appropriate numerical quadrature rule. Here, we want to apply the Gauss-Chebyshev quadrature rule. Assume that $g \in C^{N}([-1,1])$, thus

$$
\int_{-1}^{1} g(s) w(s) \mathrm{d} s \approx \frac{\pi}{N} \sum_{p=1}^{N} g\left(\cos \xi_{p}\right)
$$


where $\xi_{p}=\pi(2 p-1) / 2 N, p=1,2, \ldots, N$. It can reduce number of operations and make commodious methods to calculate the matrixes $F=\left[f_{i}\right]_{1 \leq i \leq 2^{k-1} M}$ and $\mathbf{K}=\left[K_{i j}\right]_{1 \leq i, j \leq 2^{k-1} M}$

$$
\begin{gathered}
f_{i}=\int_{0}^{1} f(x) \psi_{i}(x) w_{k}(x) \mathrm{d} x \\
\approx \frac{\sqrt{\pi} \alpha_{m}}{2^{k / 2} N} \sum_{p=1}^{N} f\left(2^{-k}\left(\cos \xi_{p}+2 n-1\right)\right) \cos \left(m \xi_{p}\right),
\end{gathered}
$$

and

$$
\begin{gathered}
K_{i j}=\int_{0}^{1} \int_{0}^{1} \psi_{i}(x) K(x, y) \psi_{j}(y) w_{k}(x) w_{k}(y) \mathrm{d} x \mathrm{~d} y \\
\approx \frac{\pi \alpha_{m} \alpha_{m^{\prime}}}{2^{k} N^{2}} \sum_{q=1}^{N} \sum_{p=1}^{N} K\left(\frac{\cos \xi_{p}+2 n-1}{2^{k}}, \frac{\cos \xi_{q}+2 n^{\prime}-1}{2^{k}}\right) \cos \left(m \xi_{p}\right) \cos \left(m^{\prime} \xi_{q}\right)
\end{gathered}
$$

We proceed by discussing the sparsity of the final system (27) which is an important issue for increasing the computation speed. But prior to that, we consider the following theorem from [15]:

Theorem 3.1.Suppose that $K_{i j}$ is the Chebyshev wavelet coefficient of the continuous kernel $K(x, y)$.If $\left|\frac{\partial^{4} K(x, y)}{\partial x^{2} \partial y^{2}}\right| \leq \delta$, where $\delta$ is a positive constant, then we have

$$
\left|K_{i j}\right| \leq \frac{\delta}{16 \pi^{4}\left(n n^{\prime}\right)^{\frac{5}{2}}\left(m m^{\prime}\right)^{2}},
$$

for $n, n^{\prime} \in \mathbf{R}$ and $m, m^{\prime} \in \mathbf{R}$.

As a conclusion from Theorem 3.1, when $i$ or $j \rightarrow \infty$ then $\left|K_{i j}\right| \rightarrow 0$ and accordingly by increasing $k$ or $M$, we can make $\mathbf{K}$ sparse. For this purpose, we choose a threshold $\varepsilon_{0}$ and define

$$
\overline{\mathbf{K}}=\left[\bar{K}_{i j}\right]_{2^{k-1} M \times 2^{k-1} M},
$$

where

$$
\bar{K}_{i j}=\left\{\begin{array}{cc}
K_{i j}, & \left|K_{i j}\right| \geq \varepsilon_{0}, \\
0, & \text { otherwise. }
\end{array}\right.
$$

Obviously, $\overline{\mathbf{K}}$ is a sparse matrix. Now, we rewrite (24) as follows

$$
U=\lambda \overline{\mathbf{K}} Z+F,
$$

and so

$$
\Psi^{t}\left(x_{j}^{\prime}\right) Z=\phi\left(x_{j}^{\prime}, \Psi^{t}\left(x_{j}^{\prime}\right)(\lambda \overline{\mathbf{K}} Z+F)\right), \quad j=1,2, \ldots, 2^{k-1} M .
$$

We can use (35) instead of (27). 


\subsection{Evaluating the matrix}

For numerical implementation of the method explained in previous part, we need to calculate matrix $\mathbf{L}=\left[L_{i j}\right]_{1 \leq i, j \leq 2^{k-1} M}$. For this purpose, by considering $i=M(n-1)+m+1$ and $\mathrm{j}=M\left(n^{\prime}-1\right)+m^{\prime}+1$, we have

$$
L_{i j}=\int_{0}^{1} \psi_{i}(y) \psi_{j}(y) \mathrm{d} y .
$$

If $n \neq n^{\prime}$ then $\psi_{i}(y) \psi_{j}(y)=0$, because their supports are disjoint, yielding $L_{i j}=0$. Hence, let $n=n^{\prime}$, by substituting $2^{k} x-2 n+1=\cos \theta$ in (36) we obtain

$$
L_{i, j}=\frac{-C_{m m^{\prime}}}{\pi} \int_{0}^{\pi} \cos m \theta \cos m^{\prime} \theta \sin \theta \mathrm{d} \theta,
$$

where

$$
C_{m m^{\prime}}= \begin{cases}1, & m=m^{\prime}=0 \\ 2, & m \neq 0 \neq m^{\prime} \\ \sqrt{2}, & \text { otherwise. }\end{cases}
$$

Now, if $\left|m+m^{\prime}\right|=1$, then

$$
\int_{0}^{\pi} \cos m \theta \cos m^{\prime} \theta \sin \theta \mathrm{d} \theta=0,
$$

implies that $\mathrm{L}_{i j}=0$ and if $\left|m+m^{\prime}\right| \neq 1$, then

$$
\begin{aligned}
L_{i j}= & \frac{C_{m m^{\prime}}}{4}\left(-\frac{\cos \left(m-m^{\prime}+1\right) \theta}{m-m^{\prime}+1}+\frac{\cos \left(-m+m^{\prime}+1\right) \theta}{-m+m^{\prime}+1}\right. \\
& \left.\left.-\frac{\cos \left(m+m^{\prime}-1\right) \theta}{m+m^{\prime}-1}+\frac{\cos \left(-m-m^{\prime}+1\right) \theta}{-m-m^{\prime}+1}\right)\right]_{0}^{\pi} .
\end{aligned}
$$

Consequently, matrix $\mathbf{L}$ has the following form

$$
\mathbf{L}=\operatorname{diag}(\underbrace{A, A, \ldots, A}_{2^{k-1} \text { times }}),
$$

where $A=\left[A_{m m^{\prime}}\right], m, m^{\prime}=0,1, \ldots, M-1$ is an $M \times M$ matrix with the following entries

$$
A_{m m^{\prime}}=\left\{\begin{array}{cc}
C_{m m^{\prime}} \frac{2\left(m^{2}+m^{\prime 2}-1\right)}{1+m^{4}-2 m^{\prime 2} m^{2}-2 m^{\prime 2}-2 m^{2}+m^{\prime 4}}, & m+m^{\prime} \text { is even }, \\
0, & m+m^{\prime} \text { is odd } .
\end{array}\right.
$$

\subsection{Error analysis}

Now, we proceed by discussing the convergence of the presented method. For this purpose, let the operators $\mathbf{K}$ and $\hat{\mathbf{T}}$ be respectively

$$
\mathbf{K} u(x)=\lambda \int_{0}^{1} K(x, y) \phi(y, u(y)) \mathrm{d} y,
$$




$$
\hat{\mathbf{T}} u(x)=\mathbf{K} u(x)+f(x),
$$

for all $u \in L_{w}^{2}[0,1]$ and $x \in[0,1]$, then Eq. (1) can be written as the operator equation

$$
u=\hat{\mathbf{T}} u \text {. }
$$

The Galerkin-Chebyshev wavelet scheme of Eq. (1) is [1,23]

$$
u_{k, M}=P_{k, M} \mathbf{K} u_{k, M}+P_{k, M} f,
$$

where $u_{k, M}$ is the approximate solution which obtained by the presented method. Now, we defined the operator $\mathbf{T}_{k, M}$ as follows

$$
\mathbf{T}_{k, M} u_{k, M}(x)=P_{k, M} \mathbf{K} u_{k, M}(x)+P_{k, M} f(x) .
$$

Therefore, Eq. (44) can be written as

$$
u_{k, M}=\mathbf{T}_{k, M} u_{k, M}
$$

In order to study the convergence of $u_{k, M}$ to $u_{0}$, where $u_{0}$ is the exact solution of Eq. (1), we first make some suitable assumptions on $K, g$ and $f$ [23].

(1) $f \in C[0,1]$.

(2) $\phi(x, y)$ is continuous on $[0,1] \times \mathbf{R}$ and there is $C_{1}>0$ s.t $\left|\phi\left(x, u_{1}\right)-\phi\left(x, u_{2}\right)\right| \leq C_{1}\left|u_{1}-u_{2}\right|$ for all $u_{1}, u_{2} \in \mathbf{R}$.

(3) There is a constant $C_{2}$ such that the partial derivative $\phi^{(0,1)}$ of $\phi$ with respect to the second variable satisfies $\left|\phi^{(0,1)}\left(x, u_{1}\right)-\phi^{(0,1)}\left(x, u_{2}\right)\right| \leq C_{1}\left|u_{1}-u_{2}\right|$ for all $u_{1}, u_{2} \in \mathbf{R}$.

(4) For $u \in C[0,1], \phi(., u()),. \phi^{(0,1)}(., u().) \in C[0,1]$.

Under the above assumptions, we can present the following theorem [30]:

Theorem 3.2. Let $u_{0} \in C[0,1]$ be solution of the equation (1). Assume that 1 is not an eigenvalue of $\mathbf{K}^{\prime}\left(u_{0}\right)$, where $\mathbf{K}^{\prime}\left(u_{0}\right)$ denote the Frechet derivative of $\mathbf{K}$ at $u_{0}$. Then the Galerkin-wavelet approximation equation (44) has, for each sufficiently large $k$, a unique solution $u_{k, M}$ in some ball of radius $\delta$ centered at $u_{0}, B\left(u_{0}, \delta\right)$. Further, there exists $0<q<1$, independent of $n$, such that if

$$
\alpha_{k, M}=\|\left(\mathbf{I}-\mathbf{T}_{k, M}^{\prime}\left(u_{0}\right)\right)^{-1}\left(\mathbf{T}_{k, M}\left(u_{0}\right)-\hat{\mathbf{T}}_{k, M}\left(u_{0}\right) \|_{\infty},\right.
$$

then

$$
\frac{\alpha_{k, M}}{1+q} \leq\left\|u_{k, M}-u_{0}\right\|_{\infty} \leq \frac{\alpha_{k, M}}{1-q}
$$

and

$$
\left\|u_{k, M}-u_{0}\right\|_{\infty} \leq C\left\|P_{k, M} u_{0}-u_{0}\right\|_{\infty}
$$

where $C$ is a constant independent of $k$ and $M$. 
Remark 2.As a conclusion from Theorems 3.2 and 2.1, if the function $u_{0}(x) \in L_{w}^{2}[0,1]$, with bounded second derivative, say $\left|u_{0}{ }_{0}(x)\right| \leq \gamma_{0}$, then the proposed method is convergence and also we have

$$
\left\|e_{k, M}\right\|_{\infty}=\left\|u_{k, M}-u_{0}\right\|_{\infty} \leq C \frac{\sqrt{\pi} \gamma}{2} \sum_{n=2^{k-1}+1}^{\infty} \sum_{m=M}^{\infty} \frac{1}{n^{\frac{5}{2}}\left(m^{2}-1\right)} .
$$

\section{Numerical examples}

In order to test the validity of the present method, two examples are solved and the numerical results are compared with their exact solution. It is seen that good agreements are achieved, as dilation parameter $a=2^{-k}$ decreases. The following norms are used for the errors of the approximation which are defined by

$$
\left\|u_{e x}-\hat{u}\right\|_{\infty}=\max \left\{\left|u_{e x}(x)-\hat{u}(x)\right|, 0 \leq x \leq 1\right\},
$$

and

$$
\left\|u_{e x}-\hat{u}\right\|_{2}=\left(\int_{0}^{1}\left|u_{e x}(x)-\hat{u}(x)\right|^{2} \mathrm{~d} x\right)^{\frac{1}{2}}
$$

where $\hat{u}(x)$ is the approximate solution of the exact solution $u_{e x}(x)$. The final nonlinear algebraic systems are solved directly by using "Fsolve" command in Maple 15 software with the Digits environment variable assigned to be 20. All calculations are run on a Pentium 4 PC Laptop with $2.50 \mathrm{GHz}$ of CPU and 4 GB of RAM.

Example 4.1.As the first example let,

$$
u(x)-\int_{0}^{1} x y \tan (u(y)) \mathrm{d} y=f(x), 0 \leq x \leq 1,
$$

where the function $f(x)$ has been so chosen that $u_{e x}(x)=\cos x$. Table 1 shows $\|e\|_{\infty}$ and $\|e\|_{2}$ at different numbers of $k, M$ and results are compared with [11].In computations, we put $\varepsilon_{0}=10^{-5}$ for $M=2$ and $\varepsilon_{0}=10^{-4}$ for $M=3$. Here, we employ the double 10-point Gauss-Chebyshev quadrature rule for numerical integration and note that increasing the number of integration points does not improve results. The approximation solution and absolute error for $k=6, M=3, \varepsilon_{0}=10^{-4}$ are shown in Figure 1 .

Table 1. Some numerical results for Example 4.1

\begin{tabular}{|c|c|c|c|c|c|}
\hline \multirow{2}{*}{$k$} & \multicolumn{2}{|c|}{$M=2$} & \multicolumn{2}{c|}{$M=3$} & \multirow{*}{*}{$\|e\|_{\infty}$} \\
& $\|e\|_{2}$ & $\|e\|_{\infty}$ & $\|e\|_{2}$ & $\|e\|_{\infty}$ & method in [11] \\
\hline 2 & $8.26 \times 10^{-3}$ & $2.23 \times 10^{-2}$ & $2.34 \times 10^{-4}$ & $1.03 \times 10^{-3}$ & $4.55 \times 10^{-2}$ \\
3 & $2.06 \times 10^{-3}$ & $5.32 \times 10^{-3}$ & $2.97 \times 10^{-5}$ & $1.21 \times 10^{-4}$ & $1.89 \times 10^{-2}$ \\
4 & $5.15 \times 10^{-4}$ & $1.41 \times 10^{-3}$ & $3.73 \times 10^{-6}$ & $1.82 \times 10^{-5}$ & $9.23 \times 10^{-3}$ \\
5 & $1.28 \times 10^{-4}$ & $3.52 \times 10^{-4}$ & $4.66 \times 10^{-7}$ & $2.32 \times 10^{-6}$ & $3.41 \times 10^{-3}$ \\
6 & $3.24 \times 10^{-5}$ & $9.81 \times 10^{-5}$ & $5.83 \times 10^{-8}$ & $2.83 \times 10^{-7}$ & $9.91 \times 10^{-4}$ \\
\hline
\end{tabular}



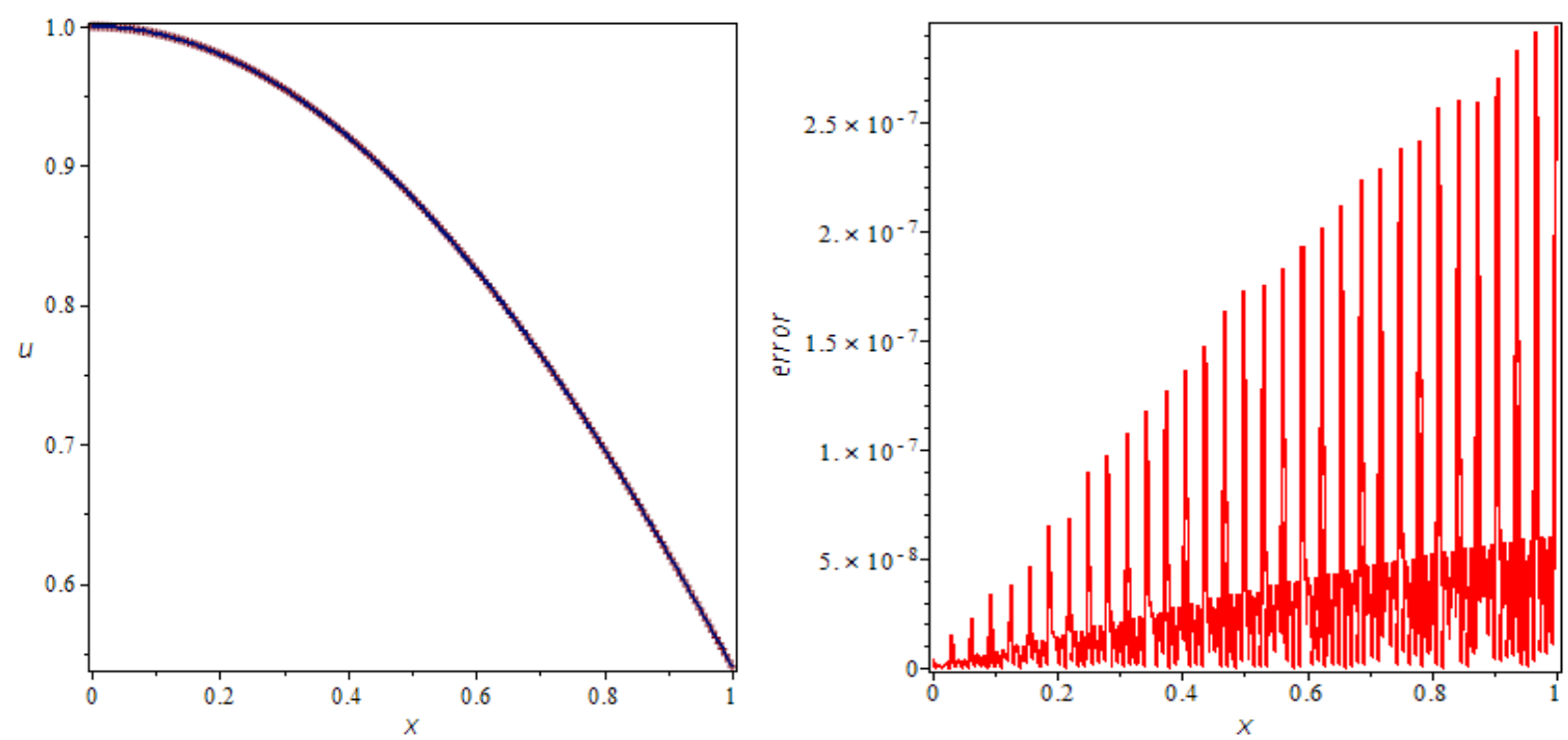

Figure 1. Approximate solution and absolute error of Example 4.1 with $k=6, M=3, \varepsilon_{0}=10^{-4}$

Example 4.2. In this example, we solve integral equation

$$
u(x)=\mathrm{e}^{x}-0.382 \sin (x)-0.301 \cos (x)+\int_{0}^{1} \sin (x+y) \ln (u(y)) \mathrm{d} y, 0 \leq x \leq 1,
$$

by the present method, in which the exact solution is $u_{e x}(x)=\mathrm{e}^{x}$. It is seen the numerical results are improved, as parameter $k$ increases. All computations performed using the 10-point Gauss-Chebyshev quadrature rule to approximate the integration numerically. Table 2 shows $\|e\|_{\infty}$ and $\|e\|_{2}$ at different numbers of $k$ and $M$ and results are compared with [11]. Here, we put $\varepsilon_{0}=10^{-5}$ for $M=2$ and $\varepsilon_{0}=10^{-4}$ for $M=3$. The approximation solution and absolute error for $k=6, M=3, \varepsilon_{0}=10^{-4}$ are shown in Figure 2.

Table 2. Some numerical results for Example 4.2

\begin{tabular}{|c|c|c|c|c|c|}
\hline \multirow[t]{2}{*}{$k$} & \multicolumn{2}{|c|}{$M=2$} & \multicolumn{2}{|c|}{$M=3$} & \multirow{2}{*}{$\begin{array}{l}\|e\|_{\infty} \\
\text { method in [11] }\end{array}$} \\
\hline & $\|e\|_{2}$ & $\|e\|_{\infty}$ & $\|e\|_{2}$ & $\|e\|_{\infty}$ & \\
\hline 2 & $1.71 \times 10^{-2}$ & $5.13 \times 10^{-2}$ & $8.05 \times 10^{-4}$ & $3.21 \times 10^{-3}$ & $3.23 \times 10^{-1}$ \\
\hline 3 & $4.34 \times 10^{-3}$ & $1.42 \times 10^{-2}$ & $1.01 \times 10^{-4}$ & $4.31 \times 10^{-4}$ & $8.07 \times 10^{-2}$ \\
\hline 4 & $1.05 \times 10^{-3}$ & $1.61 \times 10^{-3}$ & $1.27 \times 10^{-5}$ & $5.81 \times 10^{-5}$ & $3.23 \times 10^{-2}$ \\
\hline 5 & $2.76 \times 10^{-4}$ & $9.52 \times 10^{-4}$ & $1.32 \times 10^{-6}$ & $7.22 \times 10^{-6}$ & $5.61 \times 10^{-3}$ \\
\hline 6 & $3.24 \times 10^{-5}$ & $2.05 \times 10^{-4}$ & $1.99 \times 10^{-7}$ & $9.34 \times 10^{-7}$ & $1.93 \times 10^{-3}$ \\
\hline
\end{tabular}



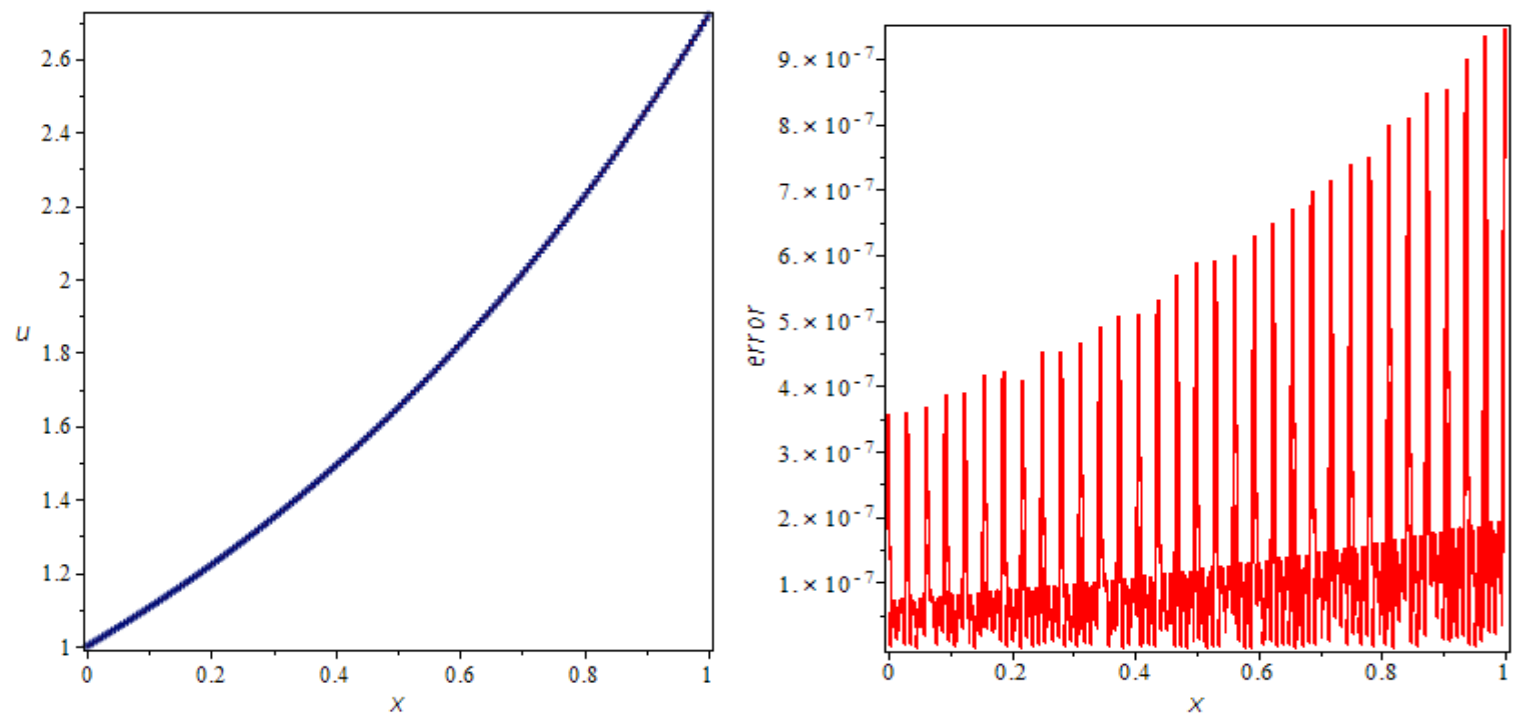

Figure 2. Approximate solution and absolute error of Example 4.2 with $k=6, M=3, \varepsilon_{0}=10^{-4}$

\section{Conclusion}

Integral equations are usually difficult to solve analytically and therefore, it is required to obtain the approximate solutions. In this study we develop an efficient and accurate method for solving nonlinear Fredholm- Hammerstein integral equations of the second kind. The properties of Chebyshev wavelets are used to reduce the problem to the solution of algebraic equations. Error analysis is provided for the new method. However, to obtain better results, using the larger parameter $k$ is recommended. The convergence accuracy of this method was examined for two numerical examples.

\section{ACKNOWLEDGEMENTS}

The authors would like to thank the editor, Dr. Reza Saadati for his comments and for managing the review process of this paper.

\section{References}

[1] H. Kaneko, R. D. Noren, B. Novaprateep, Wavelet applications to the Petrov-Galerkin method for Hammersteinequations, Appl. Numer. Math. 45 (2003) 255-273.

[2] K. E. Atkinson, The Numerical Solution of Integral Equations of the Second Kind, Cambridge University Press, 1997.

[3] K.E. Atkinson, F.A. Potra, Projection and iterated projection methods for nonlinear integral equation, SIAM J. Numer. Anal. 24 (1989) 1352-1373.

[4] Y. Ordokhani, Solution of FredholmHammerstein integral equations with WalshHybrid functions, Int. Math. Forum. 4 (2009)969-976.

[5] A. Alipanah, M. Dehghan, Numerical solution of the nonlinear Fredholm integral equations by positive definitefunctions, Appl. Math. Comput. 190 (2007) 1754-1761. 
[6] J. Rashidinia, M. Zarebnia, New approach for numerical solution of Hammerstein integral equations, Appl. Math. Comput. 185 (2007) 147-154.

[7] K. Maleknejad, K. Nedaiasl, Application of Sinc-collocation method for solving a class of nonlinear Fredholmintegral equations, Comput. Math. Appl. 62 (2011) 3292-3303.

[8] B.K. Alpert, A class of bases in $L 2$ for the sparse representation of integral operators, SIAM J. Math. Anal. 24 (1993) 246-262.

[9] H. Adibi, P. Assari, Using CAS wavelets for numerical solution of Volterra integral equations of the second kind, Dyn. Contin.Discrete Impuls.Syst., Ser.A, Math.Anal. 16 (2009) 673-685.

[10] K. Maleknejad, K. Nouria, M. NosratiSahlan, Convergence of approximate solution of nonlinear Fredholm-Hammerstein integral equations, Commun. Nonlinear. Sci. Numer. Simulat. 15 (2010) 14321443.

[11] U. Lepik, E. Tamme, Solution of nonlinear Fredholm integral equations via the Haar wavelet method, Proc. Estonian Acad. Sci. Phys. Math. 56 (2007) 17-27.

[12] H. Adibi, P. Assari, On the numerical solution of weakly singular Fredholm integral equations of the second kind using Legendre wavelets, J. Vib. Control. 17 (2011) 689-698.

[13] E. Babolian, F. Fattahzadeh, Numerical solution of differential equations by using Chebyshev wavelet operational matrix of integration, Appl. Math. Comput. 188 (2007) 417-426.

[14] E. Babolian, F. Fattahzadeh, Numerical computation method in solving integral equations by using Chebyshevwavelet operational matrix of integration, Appl. Math. Comput. 188 (2007) 1016-1022.

[15] H. Adibi, P. Assari, Chebyshev Wavelet Method for Numerical Solution of Fredholm Integral Equations of the First Kind, Math. Probl.Eng. 2010 (2010) 17 pages.

[16] S. Sohrabi, Comparison Chebyshev wavelet method with BPFs method for solving Abel's integral equation. Ain.Shams. Eng. J. 2 (2011) 249-254.

[17] J. Biazar, H. Ebrahimi, Chebyshev wavelets approach for nonlinear systems of Volterra integral equations, Comput. Math. Appl. 63 (2012) 608-616.

[18] L. Zhu, Q. Fan, Solving fractional nonlinear Fredholmintegro-differential equations by the second kind Chebyshev wavelet, Commun. Nonlinear. Sci. Numer. Simulat. 17 (2012) 2333-2341.

[19] M. Ghasemi , M. AvassoliKajani, Numerical solution of time-varying delay systems by Chebyshev wavelets. Appl. Math. Model.35 (2011) 5235-44.

[20] Y. Wang, Q. Fan. The second kind Chebyshev wavelet method for solving fractional di®erential equations, Appl. Math.Comput. 218 (2012) 8592-8601.

[21] Y. Li, Solving a nonlinear fractional differential equation using Chebyshev wavelets, Commun. Nonlinear. Sci. Numer. Simulat. 11 (2011) 2284-2292.

[22] I. Daubechies, Ten Lectures on Wavelets, SIAM/CBMS, Philadelphia PA, 1992.

[23] XuDinghua, Numerical Solutions for Nonlinear Fredholm Integral Equations of the Second Kind and Their Superconvergence, J. Shanghai. Univ. 1 (1997) 98-104.

[24] S. Kumar, I. Sloan, A new collocation-type method for Hammerstein integral equations, Math. Comput. 48 (1987) 585-593. 